\title{
3D GIS BASED EVALUATION OF THE AVAILABLE SIGHT DISTANCE TO ASSESS SAFETY OF URBAN ROADS
}

\author{
M. Bassani ${ }^{\text {a }}$, N. Grasso ${ }^{\text {a }}$, M. Piras ${ }^{\mathrm{a}, *}$ \\ ${ }^{a}$ Dept. of Environment, Land and Infrastructure Engineering, Politecnico di Torino, 24 corso Duca degli Abruzzi, Turin, 10024 Italy \\ (marco.bassani, nives.grasso, marco.piras)@polito.it
}

\section{Commission III, WG III/4}

KEY WORDS: 3D Sight Analysis, Sight Distance, Road Safety, Mobile Mapping, Digital Terrain Model, Sight Obstruction, Low-Cost Sensors, GNSS/IMU

\begin{abstract}
:
The available sight distance (ASD) in front of the driver to detect possible conflicts with unexpected obstacles is fundamental for traffic safety. In the last 20 years, road design software (RDS) has been continuously updated with dedicated modules to estimate ASD, thus assessing the quality of project from a safety point of view. Unfortunately, the evaluation of ASD still represents an issue in the case of existing road, and the object of discussion in the research community. To avoid problems related to the limitation associated with the use of digital terrain models typically employed in RDS, the Geographic Information Systems (GIS) software can use digital surface models (DSM) which are more flexible in the modelling of sight obstruction due to vegetation, street furniture, and vertical surfaces largely diffused in urbanized areas.

The paper deals with the evaluation of GIS in the estimation of ASD in a typical urban road where the density of sight obstruction along the roadside is relatively high. The work explores the case study of a collector road in the city of Turin (Italy). Results confirm the potentiality of GIS software in capturing the complex morphology of the urban environment, thus confirming that GIS could become an important analysis tool for road engineers in the field of road safety. The investigation here described is part of the Pro-VISION Project (funded in 2014 by the Regione Piemonte, Italy).
\end{abstract}

\section{INTRODUCTION}

About $75 \%$ of the accidents occur in the urban road network since this environment is affected by a high density of traffic made of different road users' (drivers, cyclists, and pedestrians) in terms of mass, size and speed. The number of conflicting points that occur where conflicting traffic flows intersect themselves is what distinguish the case of urban roads from the rural ones.

To reduce crashes and their worst consequences in terms of injuries and fatalities, road engineers are committed in protecting road users limiting vehicles' operating speeds and increasing the sight distances from the conflict points and/or potential obstacles along the driving path. According to standards and policies, the driver must have a visible space along his trajectory (called available sight distance, ASD) that she/he uses to control her/his vehicle, to avoid striking an unexpected object or other road users in the carriageway (Ministero delle Infrastrutture e Trasporti, 2001; AASHTO, 2011).

In the recent past, the evaluation of ASD has been implemented in road design software (RDS) that use digital terrain models (DTM), to design the horizontal and vertical alignments and to define the project surfaces (i.e., pavement, medians, margins and escarpments). The project model thus formed and the DTM are then analysed to derive the ASD from the most probable trajectories, which conventionally coincide with the centreline of each lane. With RDS, the evaluation of ASD is inaccurate since sight obstructions, such as vegetation and buildings, cannot be modelled and included in a DTM. In fact, the use of the digital surface models (DSM) will be better, because it includes the effective 3D model, where the buildings are included and some elements can be considered, but it depends on the DSM resolution.

RDS are more difficult to be used in the case of existing roads because it requires the regression of the real alignment, which is generally unknown, and the re-designed of project surfaces minimizing their distances from the DTM. Conversely, Geographic Information Systems (GIS) software can use DSM that are nowadays available for a large part of the urbanized areas. GIS can also incorporate every type of geospatial data as point cloud obtained by LiDAR, images, that may be used to include sight obstructions, and points or polygon (e.g they can be derived from Global Navigation Satellite System (GNSS) receivers), that can include vehicle trajectories defined as a set of 3D points (Khattak and Shamayleh, 2005; Castro et al., 2014). Moreover, GIS software has beneficiate from new tools in the field of sight analysis (Environmental Systems Research Institute, 2010). Unfortunately, GIS is not still diffused in the road designers community since it does not contains any tool to design road alignment and cross sections elements.

In 2013 the Regione Piemonte administration funded the Pro-VISION Project, whose participants were asked to cooperate in the tentative to fill the gap in knowledge and to promote the implementation of new tools in the field of road safety. The paper synthetized the results of the part of the

\footnotetext{
* Corresponding author
} 
project dedicated to the estimation of the ASD along urban roadways. The authors explored the problems encountered in the formation of a 3D model of an urban road section here considered as a case study. In particular, shape and positions of sight obstructions have been collected and implemented in a DSM already available in GIS. Trajectories of vehicles and the positions of drivers' eyes have been first reconstructed and then explored to estimate the ASD from the driver's point of view. The information has been used to generate sight profiles which are fundamental in the safety assessment of existing infrastructures.

\section{AVAILABLE SIGHT DISTANCE}

\subsection{Design standards}

According to the Italian standard (Ministero delle Infrastrutture e Trasporti, 2001), the existence of appropriate ASD along roads is of primary condition for traffic safety. The ASD is the length of the longitudinal road section that the driver can see in front of her/him without considering the influence of traffic, weather and road lighting. The distance of unobstructed vision (Figure 1) have to be compared with the distances necessary to safely perform some basic manoeuvres like the emergency stopping in front of an unexpected obstacle (i.e., stopping sight distance, SSD), overtaking a slower vehicle (i.e., passing sight distance, PSD), lane change in the carriageway at singular points such as intersections and exits ramps (i.e., change lane distance, CLD). In US, the decision sight distance (DSD) to detect unexpected or otherwise difficult-to-perceive information source or condition in a roadway environment is also compared to ASD (AASHTO, 2011).

It is worth noting that current standards impose the execution of the sight analysis to new construction only (Ministero delle Infrastrutture e dei Trasporti, 2001), but in case of re-design of an existing road it could be extended to improve safety.
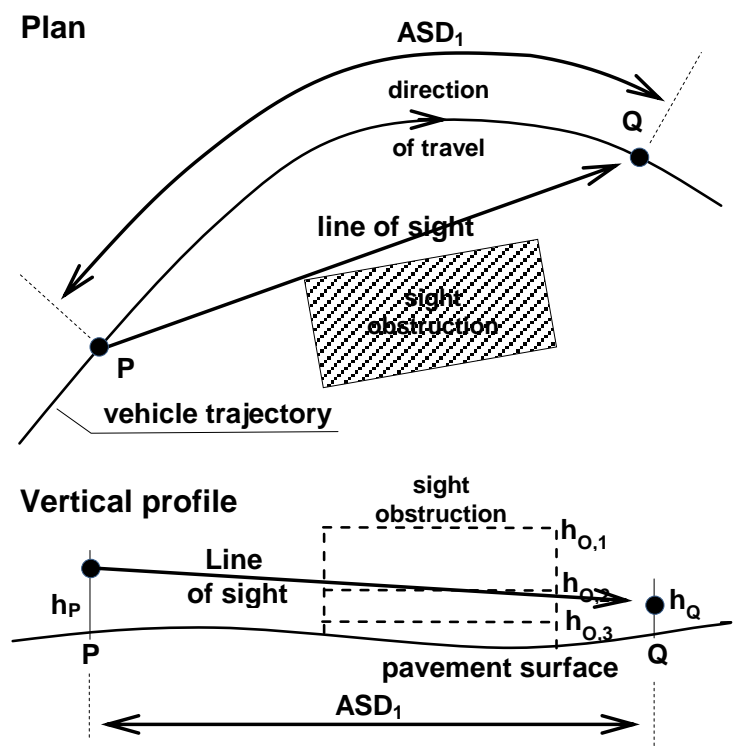

Figure 1. 3D estimation of ASD in a road sections

\subsection{ASD estimation}

Figure 1 shows the plan and the vertical profile of a typical driving situation along a horizontal curve where a driver at point $\mathrm{P}$ is able to see all the points along the trajectory to the point $\mathrm{Q}$ if the sight obstruction limits the field of vision. This is the case of sight obstructions tall enough (height equal to $\mathrm{h}_{\mathrm{O}, 1}$ ) to obscure the part of trajectory over Q (i.e., $\mathrm{ASD}=\mathrm{ASD}_{1}$ ). Conversely, small sight obstructions with a height below the line of sight $\left(\mathrm{h}_{\mathrm{O}, 3}\right)$ do not create any visual limitations, so the driver can see over the point Q (i.e., ASD > $\mathrm{ASD}_{1}$ ). In both cases, the evaluation of ASD is relatively simple and can be carried out referring to the plan view.

The case of obstruction with a height closed to the line of sight $\left(\mathrm{h}_{\mathrm{O}, 2}\right)$ cannot be analysed referring to planar representation (vertical or horizontal), and have to be investigated referring to the $3 \mathrm{D}$ analysis.

Such analysis is also necessary in case of vertical and horizontal curve combination, and when multiple sight obstructions are located along the roadside, like in the case of urban roads. In fact, the ASD is heavily affected by the road geometrics and by several sight obstructions located in the roadside and median like traffic barriers, vegetation, parked vehicles, street furniture, fences, buildings and other road elements. In night-time conditions on roads which are not provided of public lighting systems, the ASD is limited in vertical sag curves because the space illuminated by the vehicle headlights is shorter than the corresponding one along grades. According to Figure 1, ASD depends also on the height of the driver's eye above the road surface $\left(h_{P}\right)$, and the object height above the pavement surface $\left(\mathrm{h}_{\mathrm{Q}}\right)$ that road design standard establish in function of the driving manoeuvre considered (SSD, PSD, CLD, DSD, and others according to the national policy adopted). Finally, the ASD is estimated not considering the presence of other vehicles and the effects of adverse environmental conditions (i.e., fog).

As a result, in case of existing roads the estimation of ASD is possible through (a) direct field measurements, or (b) indirect measurements on a 3D model that represent the road scenario. The (a) case implies the total or partial closure of the road section to traffic for the time that is necessary to perform the survey, thus submitting the operators involved in the field to risky working conditions; furthermore, the evaluation is punctual and a lot of effort and time is necessary to get a sufficient quantity of ASD data. The (b) case imposes the acquisition and the processing of a consistent and robust quantity of geospatial data, which is nowadays possible recurring to existing database or re-creating it from field surveys. Geospatial data must include 3D elements like the pavement surface, the margins, and any potential sight obstructions (trees, buildings, street furniture, etc.). Afterwards, a relatively small effort is necessary to treat and process such data to form the 3D model. Finally, the vehicles trajectories are reconstructed and ASD can be evaluated in a greater number of points to achieve the desired level of accuracy.

\section{SPATIAL DATA ACQUISITION AND TERRAIN MODELLING}

\subsection{ProVISION project}

The main aim of the ProVISION project was to develop a set of tools to carry out visibility analysis on existing infrastructures and for any kind of road user. One of the objectives of this 
project was to implement a low-cost mobile mapping device to acquire images made of multiple sensors of mass market (e.g. GNSS, IMU, webcam and action-cam), and to provide georeferenced and spatially oriented images for photogrammetric purposes. Only the part of the investigation dedicated to vehicle users is commented in this paper.

To perform visibility analysis, a 3D model of the road environment was created integrating available data with new object surveyed in the field. As a case study, the urban road section of corso Castelfidardo in Turin (Italy) was considered as the tests site. The main steps of the Pro-VISION project are summarized as follows:

1. development of a low-cost mobile mapping system (hereafter MMS) for the acquisition of georeferenced spatial data (e.g. 3D images, trajectory, etc.);

2. integration between MMS data and aerial data (stereoimages and orthophotos) to generate an updated 3D model into the GIS environment;

3. road elements extraction (e.g. road markings, pedestrian crossing) to generate the trajectory (vehicle and obstacle) and to include the information about the road signals; and

4. definition of algorithms for the sight analysis.

In the following paragraphs describe the phases of creation of the 3D model, from initial data available, which were integrated with different techniques to generate the real model of the urban environment and the Digital Surface Model (DSM). The 3D model was built starting from the Turin's Municipal Technical Map at the scale of 1:1000 (edition 2014), the colored orthophotos at a resolution of $30 \mathrm{~cm}$ (edition 2012), and the digital terrain model (DTM) with a grid of $5 \mathrm{~m}$ of the Regione Piemonte (Italy).

\subsection{Generation of the DSM model}

From the Turin's Municipal Technical Map, a 2D model in a GIS system (coordinate system ETRF2000-UTM zone 32N) was created to enables the management of spatial data characterizing the road environment. In this kind of Technical Map, all the entities, which characterize the road infrastructure and the urban environment, are usually represented.

Subsequently, the model was integrated with the road markings by photogrammetric plotting using stereoscopic models of aerial images, by means the use of the ZMap software (Baz et al, 2008). In the first phase, all the road markings were defined by analysing the characteristics and differentiating dashed and continuous lines, punctual signals (stop and priority), pedestrian crossings and parking lanes. The final products were extracted in a dxf format to be imported in the GIS environment.

\subsection{Field surveys}

In the 3D model obtained as previously described, some elements as vegetation and street furniture could not be included because the resolution of the stereo images was too low. A real model of an urban environment needs such spatial data, that the authors solved recurring to the use of a MMS, which was mounted on a vehicle or a bike (Figure 2). These data can be used for photogrammetric plotting to define the position of the objects.

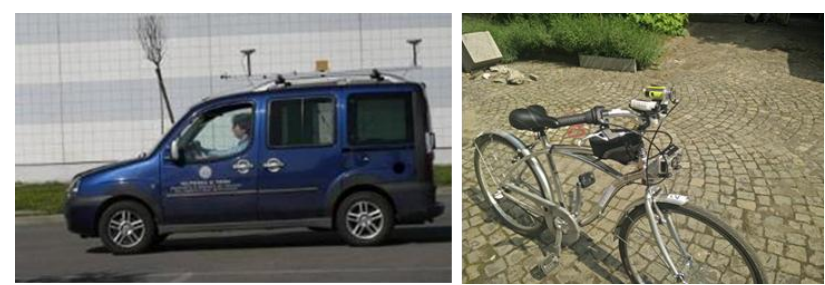

Figure 2. Mobile Mapping System on car and bicycle

In this investigation, the Authors used a specific MMS (Cina et al., 2008), which is able to acquire georeferenced data with low-cost sensors (e.g. webcam, action-cam and integrated positioning system). The image acquisition system was installed both on an instrumented vehicle with sensors of better characteristics (single and dual-frequency GNSS receiver), and on a bicycle to acquire data from bikeways (Figure 2).

On the vehicle, at the ends of the bar two webcams were connected to the PC managed by the operator inside of the car (Figure 2). Two different action-cams with GPS were also employed to obtain the driving trajectory in addition to video of the path. Finally, the GPS receiver u-blox was placed in a central position and then connected to a PC. The acquisitions were carried out along the central axis of the main carriageways in the two driving directions. One contour cam and two GoPro cams were mounted on the bike to obtain stereoscopic images of the urban environment to be implemented in the 3D model. The bike path presents in the case study was surveyed in both directions.

As well known (McGlone et al., 2004), each optical device has radial and tangential distortion due to optical lens, which lead to have an image that is not a central projection. In order to use these images for photogrammetry, it is fundamental to estimate the radial and tangential distortion parameters. In this case, this step was realized using a specific MATLAB toolbox called "Camera Calibrator" and a checkerboard pattern, (Aicardi et al, 2014).

\subsection{Road space construction}

The couple of images collected from the MMS system was used into the modern procedures based on Structure from Motion (SfM) algorithms. This procedure allows the automatic extraction of a point cloud starting from a sequences of images with a high percentage of overlapping. In this investigation, the high quantity of images allowed the use of the SfM technology to extract a new 3D model to be integrated in the initial model, even considering street furniture, vegetation, fences, and parking lanes.

Some element were included using a specific software named "Viewer" written by the author in Fortran, which allows to correlate the frames to GPS track and allows to identify and locate in the correct spatial position visible objects within images (Ajmar et al., 2011).

To generate and complete the 3D model, the 2D model previously generated was imported in the application ArcScene, with entities such as buildings that were extruded to obtain the 3D model of the urban environment. This model was used to generate the DSM. In a first stage, a fixed height was assumed for the extruded objects. Therefore, the surveys carried out with the MMS were used to correct the odds of possible obstructions on the road infrastructure. Furthermore, the parking lane placed 
all along the roadways have been considered as occupied by cars, thus considering the worst driving conditions with sight obstructions placed close to the driving path. Cars were extruded at a height of $1.60 \mathrm{~m}$ above the ground.

The applications and the software chosen to perform the sight analysis require the conversion of data format MultiPatch as integration of the DTM. The MultiPatch is a type of geometry made of planar 3D rings and triangles, used in combination to model objects that occupy discrete area or volume in the $3 \mathrm{D}$ space. MultiPatches may represent geometric objects like spheres and cubes, or objects like buildings and trees.

\subsection{DSM generation}

The sight analysis needs an accurate and updated DSM. In this case, starting from the available regional model with $5 \mathrm{~m}$ of resolution, the models have been updated by DSM generated with SfM algorithms (Hartley and Zisserman, 2003) using aerial stereopair images. Moreover, a local 3D models has been considered, which has been defined using the georeferenced images collected with the low-cost devices, and the models has been created also with SfM procedure.

From the preliminary analysis, it was clear that such DSM was not sufficiently detailed to allow the identification of possible sight obstructions. Hence, a new DSM (Figure 3) with a grid of $10 \mathrm{~cm}$ was built converting the 3D objects from the MultiPatch format. ArcGIS® allows to perform such operation through the toolbox called "Raster Calculator", in which the difference between the original DTM and the model obtained from the conversion of MultiPatch in raster was set.

\subsection{Integration techniques and updating of the GIS model}

In order to consider the real scenario and any possible changing, an "updating" of the GIS model has to be considered. Firstly, it was investigated the possibility to integrate the model with a DSM including buildings (facades, walls, and fences) generated by aerial stereo pair images.

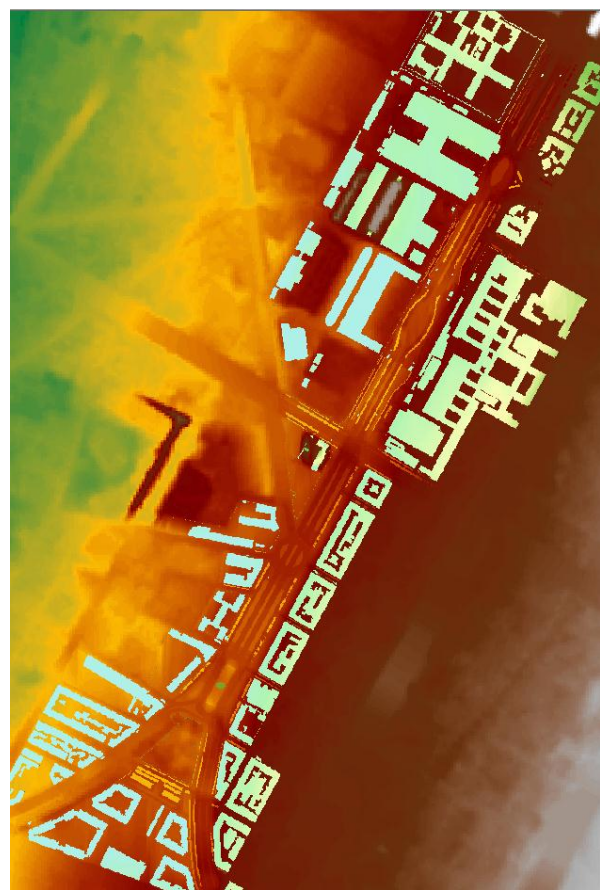

Figure 3. DSM of the test site
After the DSM generation, it was resampled to a resolution equal to $10 \mathrm{~cm}$, which is coherent with the original DSM. The union of the model was carried out using the ARCGIS ${ }^{\circledR}$ toolbox called "Mosaic" (Figure 4). In fact, when working with different DSMs, it is important to have continuity at ground level in the border of the final model. This tool allows to generate a new model from two or more raster settings, considering the overlap between the areas and a threshold tolerance. During the tests, it was decided to perform the overlap through an operator of type "maximum" (the output data generated by the overlapping of the areas is given by the maximum value among the overlapping cells), while the tolerance of mosaicking was set equal to 0 .

\subsection{Point clouds generated from images captured by smartphone}

Sometimes, it could be possible that some small or partially hidden entities that constitute obstructions in the sight analyses, are not detectable with the procedures described previously. A solution to update the existing DSM with the 3D model of the object is offered by the use of SfM approach, but using images captured by smartphone (Figure 5). In this case, the acquisition of the images requires a maximum care, considering that each point must appears in at least three images. The entire object must also be acquired from different angles and with different smartphone-object distances.

The product of these operations is a $3 \mathrm{D}$ surface that can be imported on GIS software and converted to raster files for integration with the existing DSM. To get a georeferenced model, it is possible to define coordinates (e.g. using topography) of some special points (marker) on the surface of the object. During the alignment of the images, various software allows to collimate manually the various markers and assign the reference coordinates.
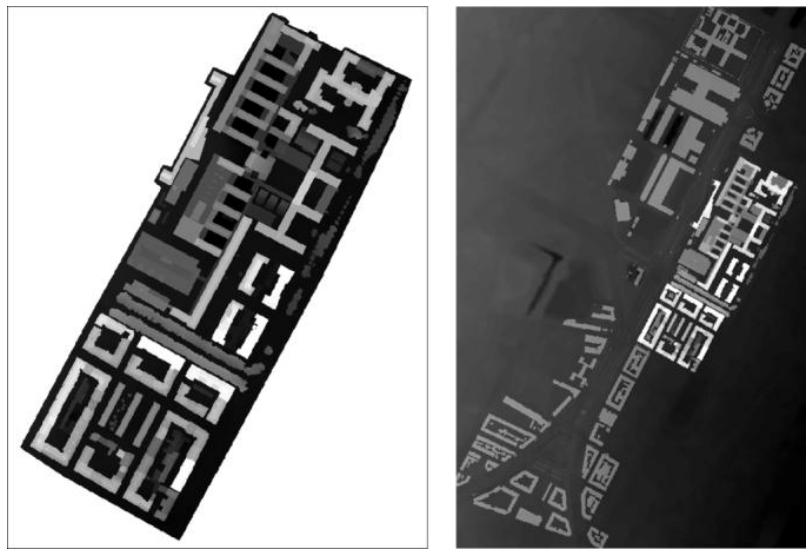

Figure 4. Example of integration DSM (grid $=20 \mathrm{~cm}$ ); its integration in the DSM of the road infrastructure
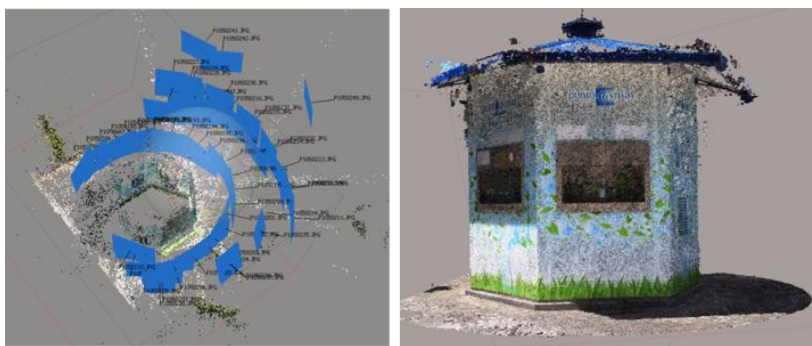

Figure 5. 3D model formation with images captured by smartphone 


\section{MODEL ANALYSIS}

The DSM built according to the procedure described in Paragraph 3 (Figure 6) was explored considering the positions of the driver's point of view according to the road standards (Ministero delle Infrastrutture e dei Trasporti, 2001) to derive the ASD considering the presence of obstructions that limit the visibility along the road.

To pursue such objective, in the GIS environment a specific toolbox that allows the use in sequence of several geoprocessing tools was formulated and implemented with the ArcGIS ${ }^{\circledR}$ ModelBuilder . The main advantage in using the ModelBuilder is that the operations can be automated. In fact, the process is first saved and then run whenever it being necessary, or change it by modifying the input parameters to produce new results. As a result, the ModelBuilder allows setting models with a complex workflow replicable through a sequences of geoprocessing tools, where the output of the first becomes the input of the following one. The instruction can be dragged on the main screen where it is asked to indicate the data input and the output. The toolbox can be used both for 2- or 3D analyses.

ArcGIS ${ }^{\circledR}$ has several applications dedicated to the spatial analysis; between them, "Line of Sight" has been specifically develop to support the analysis of visibility. The first instruction to be used in the "Line of Sight" application is named "Construct Sight Line" that connect the point of observation to the target with a line. Working in a GIS environment, these data are organized in a georeferenced database and in order to create a unique connection between an observation point and the corresponding target identified as ObjectID, these database are related precisely through field which identify the entities themselves.

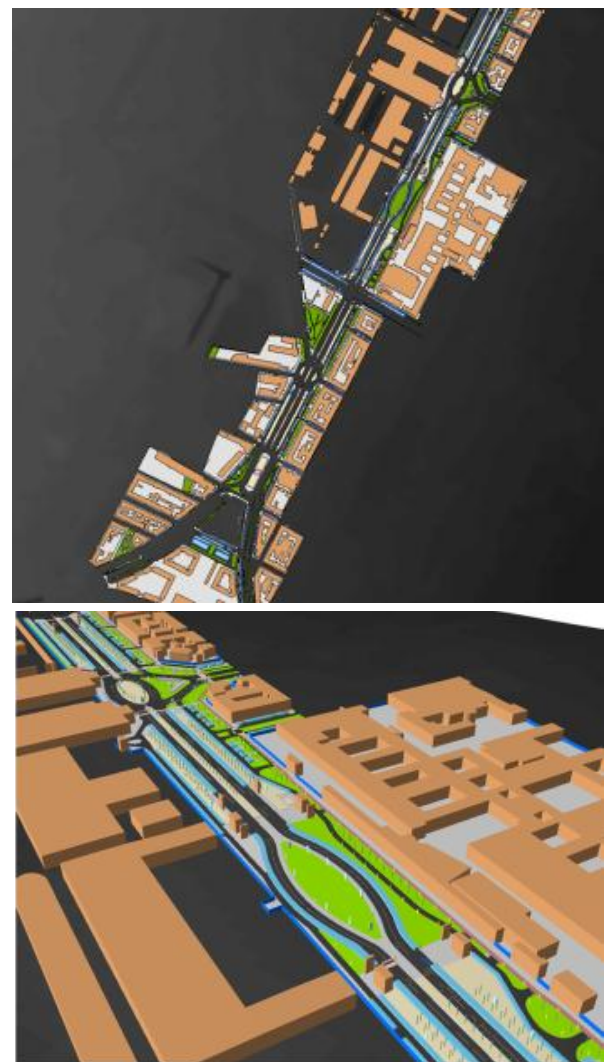

Figure 6. 2D and 3D GIS model of the test site
The sight lines obtained become the input of the second tool, which analyses and evaluates any spatial intersections with the DSM. The products of this second process are the lines of sight (Figure 7), which in the interface of the software assume a green color when they do not intersect the DSM, and red as long as they intersect a sight obstruction as part of the DSM. The coordinates of the point of obstruction constitute the second output, to which the $3 \mathrm{D}$ coordinates are evaluated with the command Add XY Coordinates.

Figure 7 shows the procedure adopted in the estimation of the ASD along the portion of the model included in Figure 8. The driving trajectory was assumed in the centre of the lane. The points of observation ( $\mathrm{P}$ point in Figure 7) was then placed at $1.1 \mathrm{~m}$ above the road surface; a step of $50 \mathrm{~m}$ along the driving path was assumed to investigate the ASD. Figure 8 shows the position on the model of the eight points considered in the sight analysis.

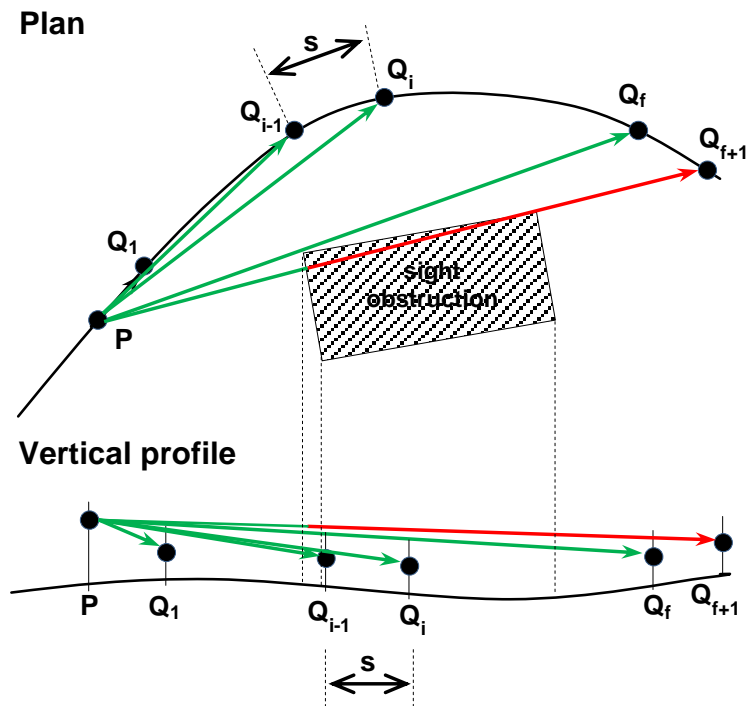

Figure 7. Representation of the algorithm used for the estimation of ASD

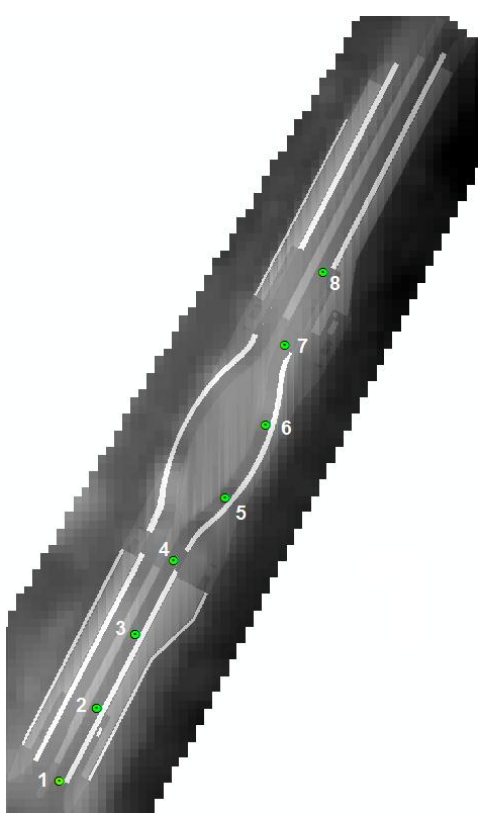

Figure 8. DSM model of the case study with eight points investigated for the ASD estimation 
The target point was placed along the driving path at a height of $0.10 \mathrm{~m}$ above the road surface. A step of $1 \mathrm{~m}$ ( $\mathrm{s}$ in Figure 7) was considered to evaluate the position of the more distant target point. A dedicated algorithm was created to generate the position of points $\mathrm{Q}$ of Figure 7. When the first target obstructed is identified along the driving path $\left(Q_{f+1}\right.$ in Figure 7), the algorithm selects the point $\mathrm{Q}_{\mathrm{f}}$ as the more distant target visible from point $P$. Finally, all the data were collected and exported to an Excel file.

Figure 9 reports the synthesis of the results of ASD evaluation. Results shows the effects of sight limitations due to road elements (curbs, road furniture) and most of all the vehicles parked along the roadside. Minimum values of ASD where observed in points 4 and 6 which are placed closed to the shortest horizontal curves along the driving path (respectively equal to 31 and $45 \mathrm{~m}$ ). According to the Italian standards (Ministero delle Infrastrutture e Trasporti, 2001), at a design speed of $60 \mathrm{~km} / \mathrm{h}$, which is appropriate for the specific case study, the stopping sight distance (SSD) on wet pavement is equal to $70 \mathrm{~m}$, therefore ASD < SSD. To solve the sight limitation, the dismissal of the parking lane along the section indicated in Figure 9 is here proposed.

\section{CONCLUSIONS}

Sight analysis aims to estimate the space visible to the driver (available sight distance, ASD) which needs to be detected in advance to avoid limitations due to the presence of possible obstruction along the route. The existence of an ASD greater than the distance necessary to perform specific manoeuvres is a pre-requisite to create safe driving condition in a harmonious road environment.

The procedure described in the paper leads to the creation of a $3 \mathrm{D}$ model that was explored and investigated in the GIS environment. Such model was able to give back the necessary information about ASD, which can be further used to improve the safety of existing infrastructure. When the comparison of ASD with the sight distances that need to be satisfied is negative, the sight obstruction can be removed or replaced to increase the ASD and satisfy the safety needs. In this investigation, the integration of different data formats required the use of several software, which certainly affects the DSM quality with a consequent reduction in accuracy. This is the reason why in the future the authors intend to develop an automatic procedure to implement the $3 \mathrm{D}$ model to be used in the ASD analysis.

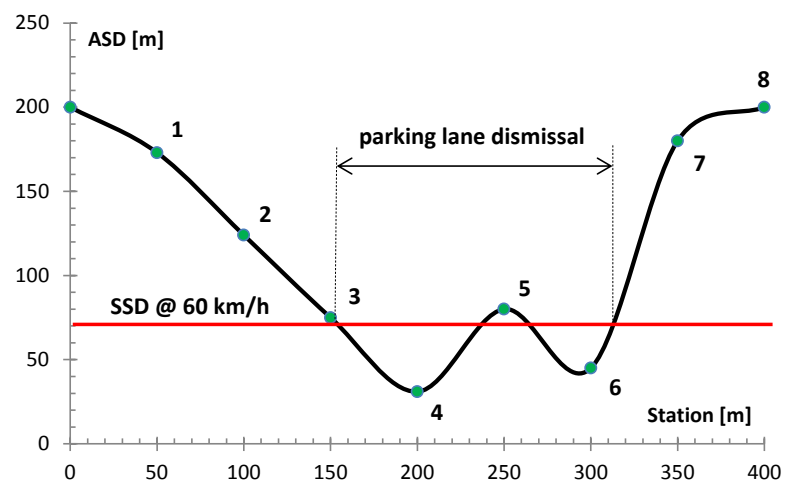

Figure 9. ASD evaluated for the case study, and comparison with SSD @ $60 \mathrm{~km} / \mathrm{h}$
Part of future investigation will be dedicated to a greater use of data acquired by mass-market instruments, like smartphone, webcam, action-cam and low-cost GNSS receiver. To take into account the fact that the urban scenario develops continuously, the idea is to create a dynamic model in which dedicated algorithms will allow the extraction of information from the recognition of geometric samples within the image and to the encoding of signals or symbols, in particular street horizontal markings and vertical signals. Finally, future research activities will be addressed to the evaluation of the ASD in correspondence to intersections, which are always affected by higher accident frequencies than longitudinal road sections.

\section{ACKNOWLEDGEMENTS}

The authors thank the "ICT- Poli di Innovazione Regione Piemonte" and FINPIEMONTE who funded the Pro-VISION Project. The other project's partner, Beanet s.r.l. and Synarea s.r.l., which have cooperated with the authors, are greatly acknowledged.

\section{REFERENCES}

Aicardi I., Lingua A., Piras M., 2014. Evaluation of Mass Market Devices for the Documentation of the Cultural Heritage. Inter. Archives of Photogrammetry, Remote Sensing and Spatial Information Sciences, XL-5, pp. 17-22.

Ajmar A., Balbo S., Boccardo P., Giulio Tonolo F., Piras M., Princic, 2011. A Low-Cost Mobile Mapping System (LCMMS) for Field Data Acquisition: a Potential Use to Validate Aerial/Satellite Building Damage Assessment. International Journal of Digital Earth, 1(21), pp. 1-21

American Association of State Highway and Transportation Officials, 2011. A Policy on Geometric Design of Highways and Streets. $4^{\text {th }}$ edition, ISBN: 1-56051-156-7.

Automobil Club Italia, Istituto Nazionale di Statistica, 2014. Road Accidents in Italy in the Year 2013. http://www.aci.it/fileadmin/documenti/studi_e_ricerche/dati_sta tistiche/incidenti/Road_accidents_in_Italy_2013.pdf (June 10, 2015).

Bassani, M., Lingua, A., Piras, M., De Agostino, M., Marinelli, G. and G. Petrini, 2012. Alignment Data Collection of Highways using Mobile Mapping and Image Analysis Techniques. Transportation Research Board of the National Academies, 91st Annual Meeting, No. 12-0312, Washington D.C.

Baz, I., Kersten, T., Büyüksalih, G., \& Jacobsen, K., 2008. Documentation of Istanbul Historic Peninsula by Static and Mobile Terrestrial Laser Scanning. The International Archives of the Photogrammetry, Remote Sensing and Spatial Information Sciences, Beijing, Vol. XXXVII. Part B 5.

Castro, M., Anta, J.A., Iglesias, L., Sánchez, J.A., 2014. GIS-Based System for Sight Distance Analysis of Highways. Journal of Computing in Civil Engineering, 28(3), pp. 04014005.

Cina, A., Lingua, A., Piras, M., 2008. Low-Cost Mobile Mapping Systems: an Italian experience. IEEE/ION Position Location and Navigation Symposium. Monterey, California, May 5-8, pp. 1033-1045. 
Environmental Systems Research Institute, 2010. ArcObjects library reference, http://edndoc.esri.com/arcobjects/9.2/ComponentHelp/esriGeo Database/ISurface_GetLineOfSight.htm (accessed July 6th, 2015).

Hartley, R., Zisserman, A., 2003. Multiple View Geometry in Computer Vision. Cambridge University Press. ISBN 0-521-54051-8.

Karara, H.M., 1989. Non-topographic Photogrammetry. American Society for Photogrammetry and Remote Sensing, 2nd edition, ISBN 0-944426-10-7.
McGlone, J.C., Mikhail, E., Bethel, J., 2004. Manual of Photogrammetry, American Society for Photogrammetry and Remote Sensing, $5^{\text {th }}$ edition, Bethesda, MD.

Ministero delle Infrastrutture e dei Trasporti, 2001. Norme Funzionali e Geometriche per la Costruzione delle Strade. Decreto Ministeriale 6792, Roma, Italia. 